\title{
Effective Capacity Channel Model for Frequency-selective Fading Channels
}

\author{
Dapeng $\mathrm{Wu}^{*} \quad$ Rohit $\mathrm{Negi}^{\dagger}$
}

\begin{abstract}
To efficiently support quality of service (QoS) in future wireless networks, it is important to model a wireless channel in terms of connection-level QoS metrics such as data rate, delay and delay-violation probability. To achieve this, in [7], we proposed and developed a link-layer channel model termed effective capacity (EC) for flat fading channels. In this paper, we apply the effective capacity technique to modeling frequency selective fading channels. Specifically, we utilize the duality between the distribution of a queue with superposition of $N$ i.i.d. sources, and the distribution of a queue with a frequency-selective fading channel that consists of $N$ i.i.d. sub-channels, to model a frequency selective fading channel. In the proposed model, a frequency selective fading channel is modeled by three EC functions; we also propose a simple and efficient algorithm to estimate these EC functions. Simulation results show that the actual QoS metric is closely approximated by the QoS metric predicted by the proposed EC channel model. The accuracy of the prediction using our model can translate into efficiency in admission control and resource reservation.

*Please direct all correspondence to Prof. Dapeng Wu, University of Florida, Dept. of Electrical \& Computer Engineering, P.O.Box 116130, Gainesville, FL 32611, USA. Tel. (352) 392-4954, Fax (352) 3920044, Email: wu@ece.ufl.edu. URL: http://www.wu.ece.ufl.edu.

${ }^{\dagger}$ Carnegie Mellon University, Dept. of Electrical \& Computer Engineering, 5000 Forbes Avenue, Pittsburgh, PA 15213, USA. Tel. (412) 268-6264, Fax (412) 268-2860, Email: negi@ece.cmu.edu. URL: http://www.ece.cmu.edu/ negi.
\end{abstract}


Key Words: Wireless channel model, QoS, delay, frequency-selective fading, large deviations theory. 


\section{Introduction}

Future cellular wireless networks are expected to support multimedia traffic with diverse QoS guarantees. Wireless channels are characterized by time-varying capacity due to channel fading; to provide explicit QoS guarantees such as a data rate, delay bound, and delaybound violation probability triplet, it is necessary to analyze a QoS provisioning system in terms of these QoS measures, for the fading channels. This task requires characterization of the service (channel modeling), and queueing analysis of the system. Specifically, a general methodology of designing QoS provisioning mechanisms at a wireless network node, involves four steps:

1. Channel measurement: e.g., measure the channel capacity process [4]. It requires channel estimation at the receiver side and feedback of channel estimates to the transmitter.

2. Channel modeling: e.g., use a Markov-modulated Poisson process to model the channel capacity process [4].

3. Deriving QoS measures: e.g., analyze the queue of the system and derive the delay distribution, given the Markov-modulated Poisson process as the service model and assuming a certain Markovian traffic model [4].

4. Relating the control parameters of QoS provisioning mechanisms to the derived QoS measures: e.g., relate the amount of allocated resource to the QoS measures. If such a relationship is known, given the QoS requirements specified by a user, we can calculate how much resource needs to be allocated to satisfy the QoS.

Steps 1 to 3 are intended to analyze the QoS provisioning mechanisms, whereas step 4 is aimed at designing the QoS provisioning mechanisms.

However, the main obstacle of applying the four steps in QoS provisioning, is the high complexity in characterizing the relation between the control parameters and the calculated QoS measures, based on existing channel models, i.e., physical-layer channel models (see Fig. 1). This is because the physical-layer channel models (e.g., Rayleigh fading model with 


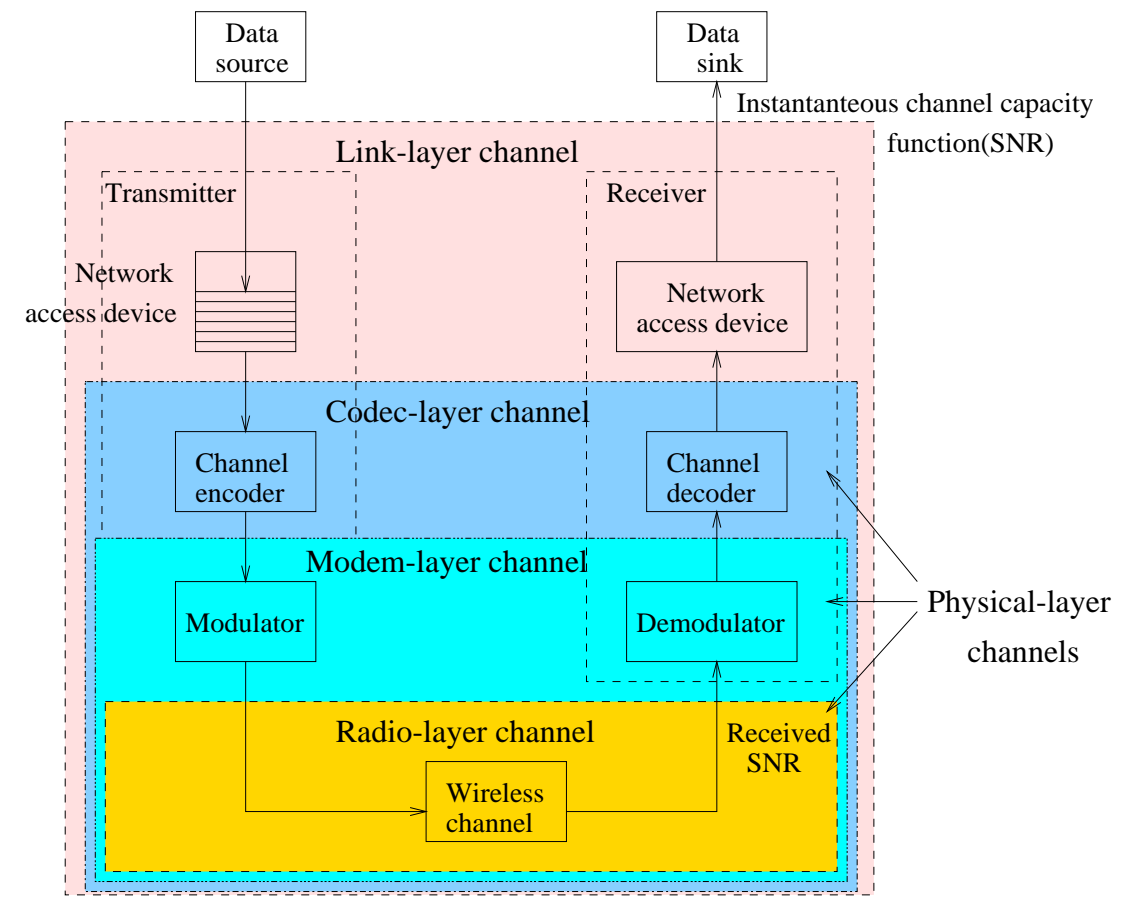

Figure 1: A wireless communication system and associated channel models.

a specified Doppler spectrum) do not explicitly characterize a wireless channel in terms of the link-level QoS metrics specified by users, such as data rate, delay and delay-violation probability. To use the physical-layer channel models for QoS support, we first need to estimate the parameters for the channel model, and then extract the link-level QoS metrics from the model. This two-step approach is obviously complex, and may lead to inaccuracies due to possible approximations in extracting QoS metrics from the models. For example, in [10, pp. 123-125], we showed that using a finite-state Markov chain channel model, one could analyze the queue (having a complexity that is exponential in the number of users) to determine what percentage of the channel resource need be allocated to a scheduler, so that a specified QoS can be satisfied; to make matters worse, the queueing analysis does not result in a closed-form relation between the control parameters and the QoS measures.

Recognizing that the limitation of physical-layer channel models in QoS support, is the difficulty in analyzing queues using them, in [7], we proposed moving the channel model up the protocol stack, from the physical-layer to the link-layer. We call the resulting model an effective capacity (EC) channel model [7], because it captures a generalized link-level capacity 
notion of the fading channel. Figure 1 illustrates the difference between the conventional physical-layer channels and the link-layer channel. In [7], we presented the EC model for flat fading channels; in this paper, we consider an EC model for frequency-selective fading channels.

To summarize, the effective capacity channel model that we propose, aims to characterize wireless channels in terms of functions that can be easily mapped to link-level QoS metrics, such as delay-bound violation probability. Furthermore, we propose a channel estimation algorithm that allows practical and accurate measurements of the effective capacity model functions. The EC model captures the effect of channel fading on the queueing behavior of the link, using a computationally simple yet accurate model, and thus, is a critical tool for designing efficient QoS provisioning mechanisms as shown in [8, 9].

The remainder of this paper is organized as follows. In Section 2, we describe various classes of wireless channel models to provide the reader with a big picture. Section 3 presents an effective capacity model for frequency selective fading channels. In Section 4, we show simulation results that demonstrate the accuracy of the proposed channel model. Section 5 concludes the paper.

\section{Wireless Channel Models}

This section describes various classes of wireless channel models.

Figure 1 shows a wireless communication system. The data source generates packets and the packets are first put into a buffer to accommodate the mismatch between the source rate and the time-varying wireless channel capacity. Then the packets traverse a channel encoder, a modulator, a wireless channel, a demodulator, a channel decoder, a network access device, and finally reach the data sink. As shown in Figure 1, one can model the communication channel at different layers as below

- Radio-layer channel: is the part between the output of the modulator and the input of the demodulator. 


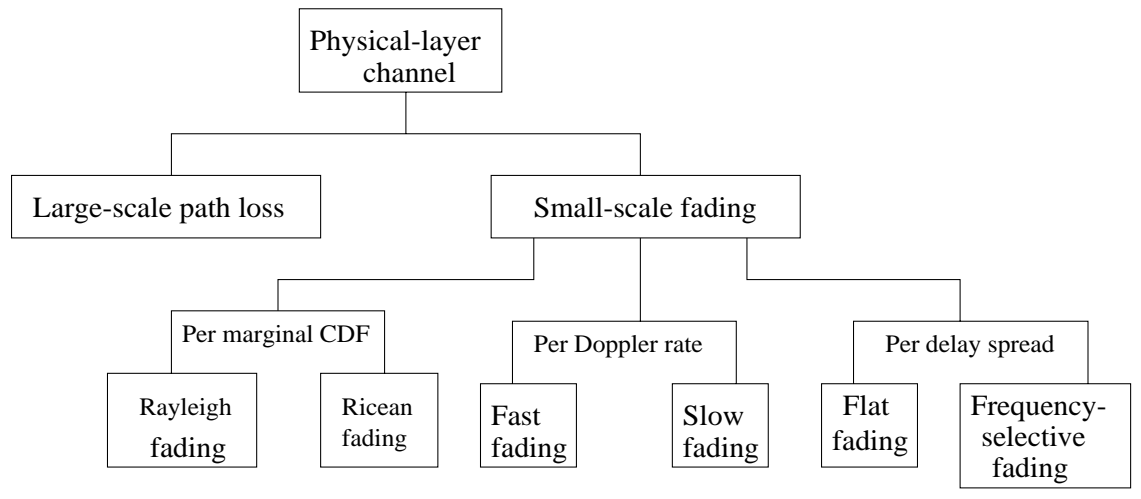

Figure 2: Classification of physical-layer channel models.

- Modem-layer channel: is the part between the output of the channel encoder and the input of the channel decoder.

- Codec-layer channel: is the part between the output of the network access device at the transmitter, and the input of the network access device at the receiver.

- Link-layer channel: is the part between the output of the data source and the input of the data sink.

The above radio-layer, the modem-layer, and the codec-layer channels can all be regarded as physical-layer channels.

As shown in Figure 2, radio-layer channel models can be classified into two categories: large-scale path loss and small-scale fading. Large-scale path loss models, also called propagation models, characterize signal strength over large transmitter-receiver separation distance (several hundreds or thousands of meters). These models specify signal attenuation as a function of distance, which is affected by prominent terrain contours (buildings, hills, forests, etc.) between the transmitter and the receiver. Path loss models describe the mean signal attenuation vs. distance in a deterministic fashion (e.g., $n$ th-power law [5]), and also the statistical variation about the mean (e.g., log-normal distribution [5]).

Small-scale fading models describe the dramatic changes in signal amplitude and phase that can be experienced as a result of small changes (as small as a half-wavelength) in the spatial separation between the receiver and the transmitter [6]. Small-scale fading can be 
slow or fast, depending on the Doppler rate. Small-scale fading can also be flat or frequencyselective, depending on the delay spread of the channel. The statistical time-varying nature of the envelope of a flat-fading signal is characterized by distributions such as Rayleigh, Ricean, Nakagami, etc. [5]. Uncorrelated scattering is often assumed, to extend these distributions to the frequency-selective case. The large-scale path loss and small-scale fading together characterize the received signal power over a wide range of distances.

A modem-layer channel can be modeled by a finite-state Markov chain [11], whose states are characterized by different bit error rates (BER). For example, in [11], a Rayleigh fading with certain Doppler spectrum is converted to a BER process, modeled by a finite-state Markov chain. The idea is the following: 1) quantize the continuous Rayleigh random variable into a discrete random variable, based on certain optimal criterion (e.g., minimum mean squared error), 2) map the resulting discrete random variable or SNR to discrete BER, for a given modulation scheme (say, binary phase shift keying), and 3) estimate the state transition probabilities, which reflect the Doppler spectrum. This procedure gives the states (i.e., BER's) and the transition probability matrix of the Markov chain.

A codec-layer channel can also be modeled by a finite-state Markov chain, whose states can be characterized by different data-rates [4], or symbol being error-free/in-error, or channel being good/bad [12]. The two state Markov chain model with good/bad states [12] is widely used in analyzing the performance of upper layer protocols such as TCP [13]. If the decoder uses hard decisions from the demodulator/detector, a codec-layer channel model can be easily obtained from a modem-layer channel model. For example, the good/bad channel model can be derived from a finite-state Markov chain with BER's as the states in the following way: first compute symbol error probability from BER; then decide the channel being good if the symbol error probability is less than a preset threshold, otherwise decide the channel being bad. The resulting good/bad channel process is a two state Markov chain.

Radio-layer channel models provide a quick estimate of the performance of wireless communications systems (e.g., symbol error rate vs. signal-to-noise ratio (SNR)). However, radio-layer channel models cannot be easily translated into complex QoS guarantees for a connection, such as bounds on delay violation probability and packet loss ratio. The rea- 
son is that, these complex QoS requirements need an analysis of the queueing behavior of the connection, which is hard to extract from radio-layer models [7]. Thus it is hard to use radio-layer models in QoS support mechanisms, such as admission control and resource reservation.

Finite-state Markov chain models for a modem-layer or codec-layer channel also require a queueing analysis of very high complexity to obtain connection-level QoS such as a data rate, delay bound, and delay-bound violation probability triplet. We showed this high complexity through an example in [10, pp. 123-125].

Recognizing that the limitation of the physical-layer channel models in QoS support, is the difficulty in analyzing queues, we propose moving the channel model up the protocol stack, from the physical-layer to the link-layer. The resulting link-layer channel model is called effective capacity model, which was developed in [7]. For convenience, we briefly describe it in the following section.

\subsection{Effective Capacity Model for Flat Fading Channels}

We first formally define statistical QoS, which characterizes the requirement of a user. First, consider a single-connection system with a flat fading channel. Assume that the user source has a fixed rate $r_{s}$ and a specified delay bound $D_{\max }$, and requires that the delay-bound violation probability is not greater than a certain value $\varepsilon$, that is,

$$
\operatorname{Pr}\left\{D(\infty)>D_{\max }\right\} \leq \varepsilon
$$

where $D(\infty)$ is the steady-state delay experienced by a flow, and $\operatorname{Pr}\left\{D(\infty)>D_{\text {max }}\right\}$ is the probability of $D(\infty)$ exceeding a delay bound $D_{\max }$. Then, we say that the user is specified by the (statistical) QoS triplet $\left\{r_{s}, D_{\max }, \varepsilon\right\}$. Even for this simple case, it is not immediately obvious as to which QoS triplets are feasible, for the given channel, since a rather complex queueing system (with an arbitrary channel capacity process) will need to be analyzed. The key contribution of [7] was to introduce a concept of statistical delay-constrained capacity termed effective capacity, which allows us to obtain a simple and efficient test, to check the feasibility of QoS triplets for a given time-varying channel. That paper dealt with flat fading 


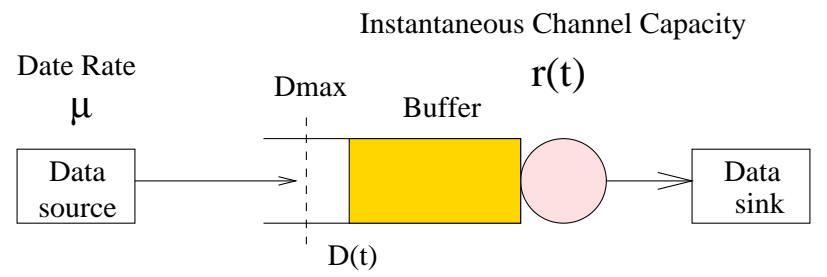

Figure 3: A queueing system model.

channels instead of frequency selective fading channels, which we consider in this paper.

Next, we briefly explain the concept of effective capacity, and refer the reader to [7] for details.

Let $r(t)$ be the instantaneous channel capacity at time $t$. Assume that, the asymptotic $\log$-moment generation function of $r(t)$

$$
\Lambda(-u)=\lim _{t \rightarrow \infty} \frac{1}{t} \log E\left[e^{-u \int_{0}^{t} r(\tau) \mathrm{d} \tau}\right]
$$

exists for all $u \geq 0$. Then, the effective capacity function of $r(t)$ is defined as

$$
\alpha(u)=\frac{-\Lambda(-u)}{u} \quad, \forall u>0 .
$$

That is,

$$
\alpha(u)=-\lim _{t \rightarrow \infty} \frac{1}{u t} \log E\left[e^{-u \int_{0}^{t} r(\tau) \mathrm{d} \tau}\right], \forall u>0 .
$$

Consider a queue of infinite buffer size supplied by a data source of constant data rate $\mu$ (see Fig. 3). It can be shown [7] that if $\alpha(u)$ indeed exists (e.g., for ergodic, stationary, Markovian $r(t)$ ), then the probability of $D(\infty)$ exceeding a delay bound $D_{\max }$ satisfies

$$
\operatorname{Pr}\left\{D(\infty)>D_{\max }\right\} \approx \gamma(\mu) e^{-\theta(\mu) D_{\max }},
$$

where $\{\gamma(\mu), \theta(\mu)\}$ are functions of source rate $\mu$ and they depend only on the channel capacity process $r(t)$. $\{\gamma(\mu), \theta(\mu)\}$ can be considered as a channel model that models the channel at the link layer (in contrast to physical layer models). The approximation (5) is accurate for large $D_{\max }$. 
In terms of the effective capacity function (4) defined earlier, the QoS exponent function $\theta(\mu)$ can be written as $[7]$

$$
\theta(\mu)=\mu \alpha^{-1}(\mu)
$$

where $\alpha^{-1}(\cdot)$ is the inverse function of $\alpha(u)$. Once $\{\gamma(\mu), \theta(\mu)\}$ have been measured for a given channel, they can be used to check the feasibility of QoS triplets. Specifically, a QoS triplet $\left\{r_{s}, D_{\max }, \varepsilon\right\}$ is feasible if $\theta\left(r_{s}\right) \geq-\log \left(\varepsilon / \gamma\left(r_{s}\right)\right) / D_{\max }$.

The above effective capacity model is for flat fading channels. Next, we extend the EC model to the case of frequency selective fading channels.

\section{Modeling for Frequency Selective Fading Channels}

In [7], we modeled the wireless channel in terms of two 'effective capacity' functions; namely, the probability of non-empty buffer $\gamma(\mu)$ and the QoS exponent $\theta(\mu)$. Furthermore, we developed a simple and efficient algorithm to estimate the EC functions $\{\gamma(\mu), \theta(\mu)\}$. For flat-fading channels (e.g., Rayleigh fading with a specified Doppler spectrum), the simulation results [7] have shown that the actual QoS metric is closely approximated by the QoS metric predicted by the EC channel model and its estimation algorithm, under various scenarios. On the other hand, for frequency selective fading channels with high degrees of frequency diversity, a refinement is useful to characterize $\gamma(\mu)$ further. The technique we use is a dual of large deviations theory for many sources.

In this section, we use the duality between the distribution of a queue with superposition of $N$ i.i.d. sources, and the distribution of a queue with a frequency-selective fading channel that consists of $N$ i.i.d. sub-channels, to propose a channel model, specified by three functions $\left\{\beta^{(c)}(\mu), \eta^{(c)}(\mu), \theta_{1}(\mu)\right\}$.

The remainder of this section is organized as follows. Section 3.1 presents large deviation results for a queue with many inputs, which provide us the method to model frequency-selective fading channels. In Section 3.2, we describe an effective capacity model for frequency-selective fading channels. 


\subsection{Large Deviation Results for a Queue with Many Inputs}

In [1], Botvich and Duffield obtained a large deviation result for the queue at a multiplexer with $N$ inputs. To state their results, let $Q^{N}(\infty)$ be the length of the steady-state queue due to a superposition of $N$ i.i.d. stationary sources, served at constant rate $N \times r$ ( $r$ fixed). Denote by $A^{N}(t)(t \geq 0)$ the amount of aggregate traffic from the $N$ sources over the time interval $[0, t)$. Assume that the many-source-asymptotic ${ }^{1} \log$-moment generating function of $A^{N}(t)$, defined as

$$
\Lambda_{t}(u)=\lim _{N \rightarrow \infty} \frac{1}{N t} \log E\left[e^{u\left(A^{N}(t)-N r t\right)}\right],
$$

exists for all $u>0$ and $t>0$.

Define $\Lambda_{t}^{*}$ the Legendre-Fenchel transform of $\Lambda_{t}$, through

$$
\Lambda_{t}^{*}(B)=\sup _{u}\left(B \times u-\Lambda_{t}(u)\right)
$$

Under appropriate conditions, paralleling the result for large buffer asymptotic [10, page 75, Eq. (23)], Botvich and Duffield's result for many source asymptotic [1] is

$$
\log _{e} \operatorname{Pr}\left\{Q^{N}(\infty) \geq B\right\} \sim-N \times I(B / N) \quad \text { as } N \rightarrow \infty,
$$

where $I(B)=\inf _{t>0} t \Lambda_{t}^{*}(B / t)$, and $f(x) \sim g(x)$ means that $\lim _{x \rightarrow \infty} f(x) / g(x)=1$. Eq. (9) yields the approximation

$$
\operatorname{Pr}\left\{Q^{N}(\infty) \geq B\right\} \approx e^{-N \times I(B / N)},
$$

By introducing a prefactor $\beta^{(s)}(r)$, a more accurate approximation was proposed in [2] as below,

$$
\begin{aligned}
\operatorname{Pr}\left\{Q^{N}(\infty) \geq B\right\} & \approx \beta^{(s)}(r) \times e^{-N \times I(B / N)} \\
& \approx \beta^{(s)}(r) \times e^{-\eta^{(s)}(r) \times N} \times e^{-\theta_{B}(N, r) \times B},
\end{aligned}
$$

\footnotetext{
${ }^{1}$ Many source asymptotic is for the case where the number of sources $N$ goes to $\infty$ while large buffer asymptotic is for the case where the buffer size $B$ goes to $\infty$.
} 
where $\beta^{(s)}(r)$ and $\eta^{(s)}(r)$ are functions of $r, \theta_{B}(N, r)$ is a function of $N$ and $r$, and $\eta^{(s)}(r)$ and $\theta_{B}(N, r)$ satisfy $[1]$

$$
\lim _{B \rightarrow \infty}\left(I(B)-\theta_{B}(N, r) \times B\right)=\eta^{(s)}(r),
$$

and

$$
\eta^{(s)}(r)=-\lim _{t \rightarrow \infty} t \Lambda_{t}\left(\theta_{B}(N, r)\right)
$$

under appropriate conditions. The quantity $\beta^{(s)}(r) \times e^{-\eta^{(s)}(r) \times N}$ can be regarded as the probability that the buffer is non-empty; this probability decays exponentially as the number of sources $N$ increases.

If the quantity of interest is the steady-state delay $D^{N}(\infty)$, then the probability of $D^{N}(\infty)$ exceeding a delay bound $D_{\max }$ satisfies

$$
\operatorname{Pr}\left\{D^{N}(\infty) \geq D_{\max }\right\} \approx \beta^{(s)}(r) \times e^{-\eta^{(s)}(r) \times N} \times e^{-\theta^{(s)}(N, r) \times D_{\max }}
$$

where $\theta^{(s)}(N, r)=\theta_{B}(N, r) \times N \times r$. Thus, the triplet $\left\{\beta^{(s)}(r), \eta^{(s)}(r), \theta^{(s)}(N, r)\right\}$ models the aggregate source.

In the following section, we use the duality between traffic modeling $\left(\left\{\beta^{(s)}(r), \eta^{(s)}(r), \theta^{(s)}(N, r)\right\}\right)$ and channel modeling to propose a link-layer model for frequency selective fading channels, specified by a triplet $\left\{\beta^{(c)}(\mu), \eta^{(c)}(\mu), \theta_{N}(\mu)\right\}$. It is clear that we intend $\left\{\beta^{(c)}(\mu), \eta^{(c)}(\mu), \theta_{N}(\mu)\right\}$ to be the channel duals of the source functions $\left\{\beta^{(s)}(r), \eta^{(s)}(r), \theta^{(s)}(N, r)\right\}$. Just as the constant channel rate $r$ is used in source traffic modeling, we use the constant source traffic rate $\mu$ in modeling the channel.

\subsection{Channel Modeling}

Consider a queue of infinite buffer size supplied by a data source of constant data rate $\mu$, served by $1 / N$ fraction of a frequency-selective fading channel that consists of $N$ i.i.d. subchannels. The queue is served by $1 / N$ fraction of the channel to keep the system $\operatorname{load}^{2}$

\footnotetext{
${ }^{2}$ The system load is defined as the ratio of the expected source rate to the ergodic channel capacity.
} 
constant as $N$ increases. The large deviation results in Section 3.1 can be easily adapted to this case. The difference is that whereas in Section 3.1, the source rate was variable while the channel capacity per source was constant, in this section, the source rate is constant while the channel capacity is variable. Similar to (15), it can be shown that the probability of $D(\infty)$ exceeding a delay bound $D_{\max }$ satisfies

$$
\operatorname{Pr}\left\{D(\infty) \geq D_{\max }\right\} \approx \gamma_{N}(\mu) \times e^{-\theta_{N}(\mu) \times D_{\max }}
$$

where the functions $\left\{\gamma_{N}(\mu), \theta_{N}(\mu)\right\}$ characterize the frequency-diversity channel with $N$ independent sub-channels, and the function $\gamma_{N}(\mu)$ can be approximated by

$$
\gamma_{N}(\mu) \approx \beta^{(c)}(\mu) \times e^{-\eta^{(c)}(\mu) \times N}
$$

Assuming equality in (17), we can easily derive a method to estimate $\eta^{(c)}(\mu)$ and $\beta^{(c)}(\mu)$ as below

$$
\eta^{(c)}(\mu)=-\log \left(\gamma_{N}(\mu) / \gamma_{1}(\mu)\right) /(N-1)
$$

and

$$
\beta^{(c)}(\mu)=\gamma_{1}(\mu) \times e^{\eta^{(c)}(\mu)}
$$

where $\gamma_{1}(\mu)$ and $\gamma_{N}(\mu)$ can be estimated by Eq. (39) in the Appendix.

For the case where the sub-channels are i.i.d., a simplification occurs. Let $r_{N}(t)$ be the instantaneous channel capacity of $1 / N$ fraction of a frequency-selective fading channel with $N$ i.i.d. sub-channels, at time $t$. Then, the effective capacity function of $r_{N}(t)$ is defined as

$$
\alpha_{N}(u)=-\lim _{t \rightarrow \infty} \frac{1}{u t} \log E\left[e^{-u \int_{0}^{t} r_{N}(\tau) \mathrm{d} \tau}\right], \forall u>0
$$

if it exists. Let $r_{1}(t)$ be the instantaneous channel capacity of one sub-channel of the frequency-selective fading channel, at time $t$. Then, the effective capacity function of $r_{1}(t)$ is defined as

$$
\alpha_{1}(u)=-\lim _{t \rightarrow \infty} \frac{1}{u t} \log E\left[e^{-u \int_{0}^{t} r_{1}(\tau) \mathrm{d} \tau}\right], \forall u>0 .
$$


According to [10, page 78, Eq. (30)], the QoS exponents $\theta_{N}(\mu)$ and $\theta_{1}(\mu)$ are defined as

$$
\theta_{N}(\mu)=\mu \alpha_{N}^{-1}(\mu)
$$

and

$$
\theta_{1}(\mu)=\mu \alpha_{1}^{-1}(\mu)
$$

respectively, and the two QoS exponents have a relation specified by Proposition 1 .

Proposition 1 The QoS exponents $\theta_{N}(\mu)$ and $\theta_{1}(\mu)$ satisfy

$$
\theta_{N}(\mu)=N \times \theta_{1}(\mu)
$$

For a proof of Proposition 1, see the Appendix.

From (16), (17), and (24), we have

$$
\operatorname{Pr}\left\{D(\infty) \geq D_{\max }\right\} \approx \beta^{(c)}(\mu) \times e^{-\eta^{(c)}(\mu) \times N} \times e^{-N \times \theta_{1}(\mu) \times D_{\max }} .
$$

So, the functions $\left\{\beta^{(c)}(\mu), \eta^{(c)}(\mu), \theta_{1}(\mu)\right\}$ sufficiently characterize the QoS $\operatorname{Pr}\left\{D(\infty) \geq D_{\max }\right\}$ for a frequency-selective fading channel, consisting of arbitrary $N$ i.i.d. sub-channels. There is no need to directly estimate $\gamma_{N}(\mu)$ and $\theta_{N}(\mu)$ for arbitrary $N$, i.e., using Eqs. (39) through (42) in the Appendix.

The EC channel model for frequency-selective fading channels and its application are summarized below.

1. $\left\{\beta^{(c)}(\mu), \eta^{(c)}(\mu), \theta_{1}(\mu)\right\}$ is the EC channel model.

2. $\left\{\beta^{(c)}(\mu), \eta^{(c)}(\mu), \theta_{1}(\mu)\right\}$ can be estimated by (19), (18), and (39) through (42), respectively.

3. Given the EC channel model, the QoS $\left\{\mu, D_{\max }, \varepsilon\right\}$ can be computed by Eq. (25), where $\varepsilon=\operatorname{Pr}\left\{D(\infty) \geq D_{\max }\right\}$. 


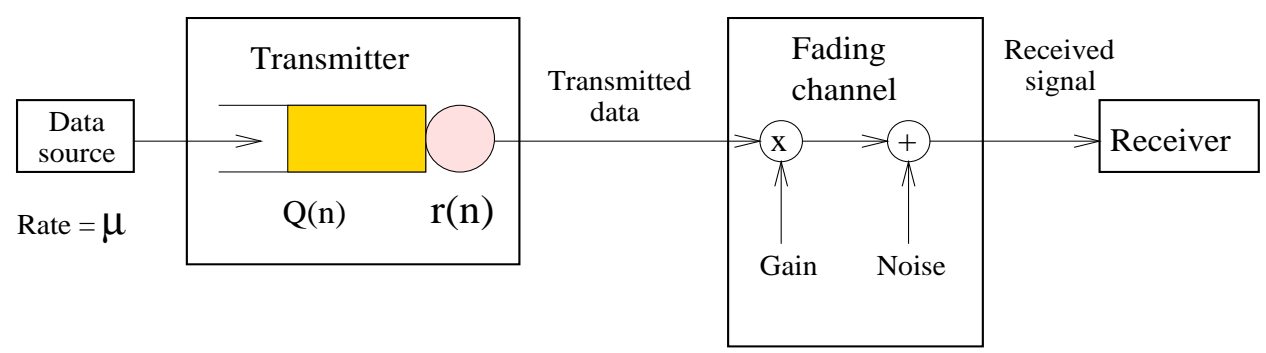

Figure 4: The queueing model used for simulations.

\section{Simulation Results}

\subsection{Simulation Setting}

We simulate the discrete-time system depicted in Figure 4. In this system, the data source generates packets at a constant rate $\mu$. Generated packets are first sent to the (infinite) buffer at the transmitter, whose queue length is $Q(n)$, where $n$ refers to the $n$-th sample-interval. The head-of-line packet in the queue is transmitted over a frequency selective fading channel at data rate $r(n)$. We use a fluid model, that is, the size of a packet is infinitesimal. In practical systems, the results presented here will have to be modified to account for finite packet sizes.

Denote $h_{i}(n)$ the channel voltage gain of sub-channel $i(i=1,2, \cdots, N)$ in a frequency selective fading channel, at sample-interval $n$. We assume that the transmitter has perfect knowledge of the current channel gains $h_{i}(n)$ of each sub-channel $i$ at each sample-interval $n$. Therefore, it can use rate-adaptive transmissions and ideal channel codes, to transmit packets without decoding errors. Thus, the transmission rate $r_{i}(n)$ of sub-channel $i$ is equal to the instantaneous (time-varying) capacity of the fading channel, as below,

$$
r_{i}(n)=B_{c} \log _{2}\left(1+\left|h_{i}(n)\right|^{2} \times P_{0} / \sigma_{n}^{2}\right)
$$

where $B_{c}$ denotes the channel bandwidth, and the transmission power $P_{0}$ and noise variance $\sigma_{n}^{2}$ are assumed to be constant.

The average SNR is fixed in each simulation run. We define $r_{\text {awgn }}$ as the capacity of an 
equivalent AWGN channel, which has the same average SNR, i.e.,

$$
r_{\text {awgn }}=B_{c} \log _{2}\left(1+S N R_{\text {avg }}\right)
$$

where $S N R_{\text {avg }}=E\left[\left|h_{i}(n)\right|^{2} \times P_{0} / \sigma^{2}\right]=P_{0} / \sigma^{2}$. We set $E\left[\left|h_{i}(n)\right|^{2}\right]=1$. Then, we can eliminate $B_{c}$ using Eqs. (26) and (27) as,

$$
r_{i}(n)=\frac{r_{a w g n} \log _{2}\left(1+\left|h_{i}(n)\right|^{2} \times S N R_{a v g}\right)}{\log _{2}\left(1+S N R_{a v g}\right)} .
$$

Since the transmission rate $r(n)$ is equal to $1 / N$ of the sum of the instantaneous capacities of the $N$ sub-channels, we have

$$
r(n)=\frac{1}{N} \sum_{i=1}^{N} r_{i}(n)=\frac{\sum_{i=1}^{N} r_{a w g n} \log _{2}\left(1+\left|h_{i}(n)\right|^{2} \times S N R_{a v g}\right)}{N \log _{2}\left(1+S N R_{a v g}\right)}
$$

The channel gain $h_{i}(n)$ of each sub-channel $i(i=1,2, \cdots, N)$ is assumed to be Rayleighdistributed and is generated by an $\mathrm{AR}(1)$ model as below,

$$
h_{i}(n)=\kappa \times h_{i}(n-1)+v_{i}(n),
$$

where $v_{i}(n)$ are i.i.d. complex Gaussian variables with zero mean and variance of $\left(1-\kappa^{2}\right) / 2$ per dimension. It is clear that (30) results in $E\left[\left|h_{i}(n)\right|^{2}\right]=1$. The coefficient $\kappa$ determines the Doppler rate, i.e., the larger the $\kappa$, the smaller the Doppler rate. Specifically, the coefficient $\kappa$ can be determined by the following procedure: 1$)$ compute the coherence time $T_{c}$ by $[5$, page 165]

$$
T_{c} \approx \frac{9}{16 \pi f_{m}}
$$

where the coherence time is defined as the time, over which the time auto-correlation function of the fading process is above $0.5 ; 2$ ) compute the coefficient $\kappa$ by $^{3}$

$$
\kappa=0.5^{T_{s} / T_{c}} .
$$

\footnotetext{
${ }^{3}$ The auto-correlation function of the $\mathrm{AR}(1)$ process is $\kappa^{m}$, where $m$ is the number of sample intervals. Solving $\kappa^{T_{c} / T_{s}}=0.5$ for $\kappa$, we obtain (32).
} 


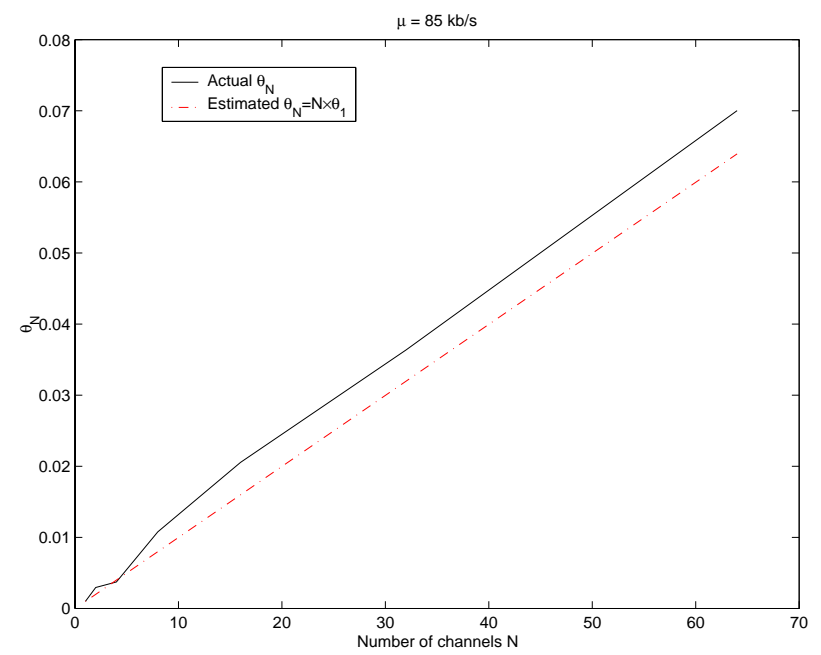

Figure 5: Actual and estimated $\theta_{N}$ vs. $N$.

In all the simulations, we fix the following parameters: $r_{\text {awgn }}=100 \mathrm{~kb} / \mathrm{s}, \kappa=0.98$, and $S N R_{\text {avg }}=0 \mathrm{~dB}$. The sample interval $T_{s}$ is set to 1 milli-second. This is not too far from reality, since 3G WCDMA systems already incorporate rate adaptation on the order of 10 milli-second [3]. Each simulation run is 1000-second long in all scenarios.

In the next section, we use simulation results to show the accuracy of the channel model $\left\{\beta^{(c)}(\mu), \eta^{(c)}(\mu), \theta_{1}(\mu)\right\}$.

\subsection{Accuracy of Channel Model $\left\{\beta^{(c)}(\mu), \eta^{(c)}(\mu), \theta_{1}(\mu)\right\}$}

In the simulations, for the purpose of comparison, we first directly estimate $\gamma_{N}$ and $\theta_{N}$ for various number of sub-channels $N$, using Eqs. (39) through (42); then we estimate $\left\{\beta^{(c)}(\mu), \eta^{(c)}(\mu), \theta_{1}(\mu)\right\}$ by (19), (18), and (39) through (42), respectively.

Figure 5 shows the actual and estimated $\theta_{N}$ vs. $N$ for $\mu=85 \mathrm{~kb} / \mathrm{s}$. The actual $\theta_{N}$ is meant to be the $\theta_{N}$ directly measured by Eqs. (39) through (42) for the case with $N$ subchannels; the estimated $\theta_{N}$ is meant to be $N \times \theta_{1}$, i.e., Eq. (24), where $\theta_{1}$ is measured by Eqs. (39) through (42) for the case with one sub-channel. The figure indicates that 1) the actual $\theta_{N}$ linearly increase with $N$, justifying the linear relation in (24), and 2) the estimated $\theta_{N}$ can serve as a rough estimate of the actual $\theta_{N}$. 


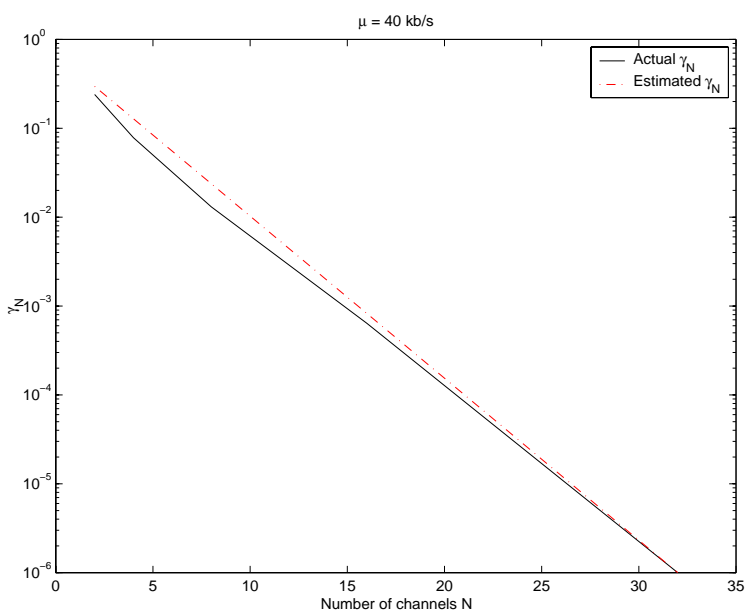

(a)

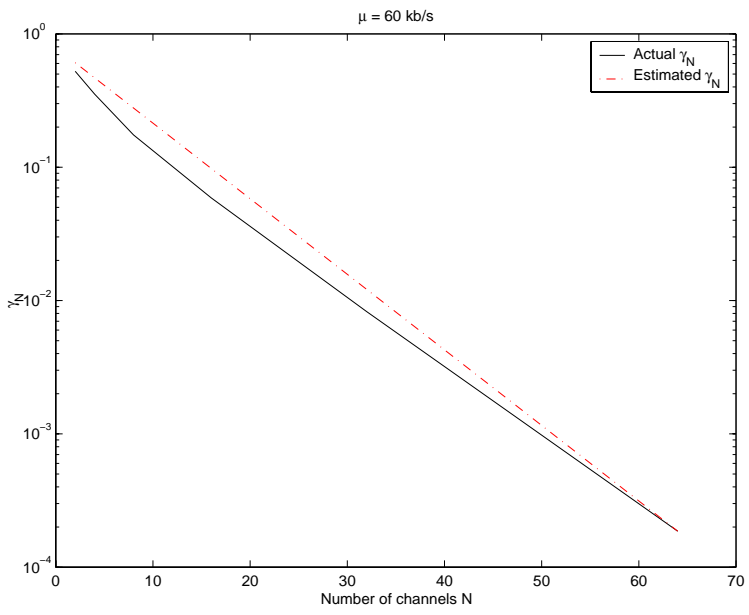

(b)

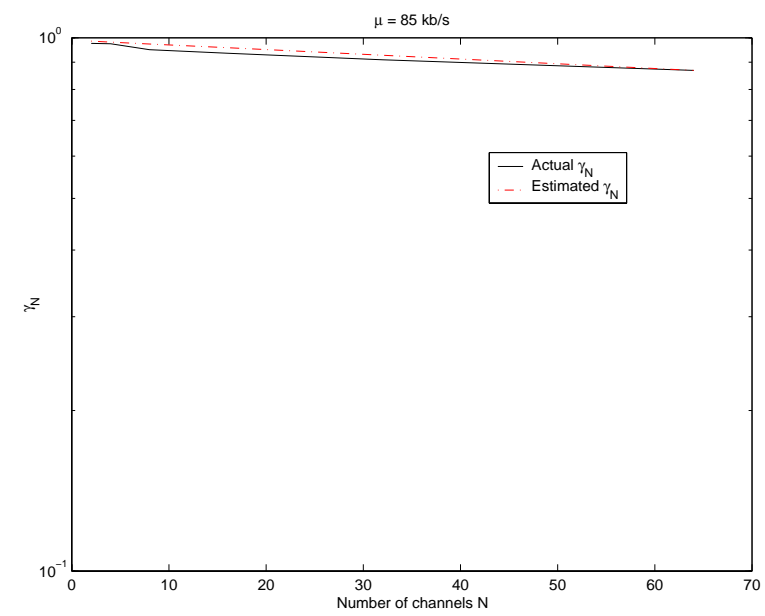

(c)

Figure 6: Actual and estimated $\gamma_{N}$ vs. $N$ : (a) $\mu=40 \mathrm{~kb} / \mathrm{s}$, (b) $\mu=60 \mathrm{~kb} / \mathrm{s}$, and (c) $\mu=85$ $\mathrm{kb} / \mathrm{s}$. 


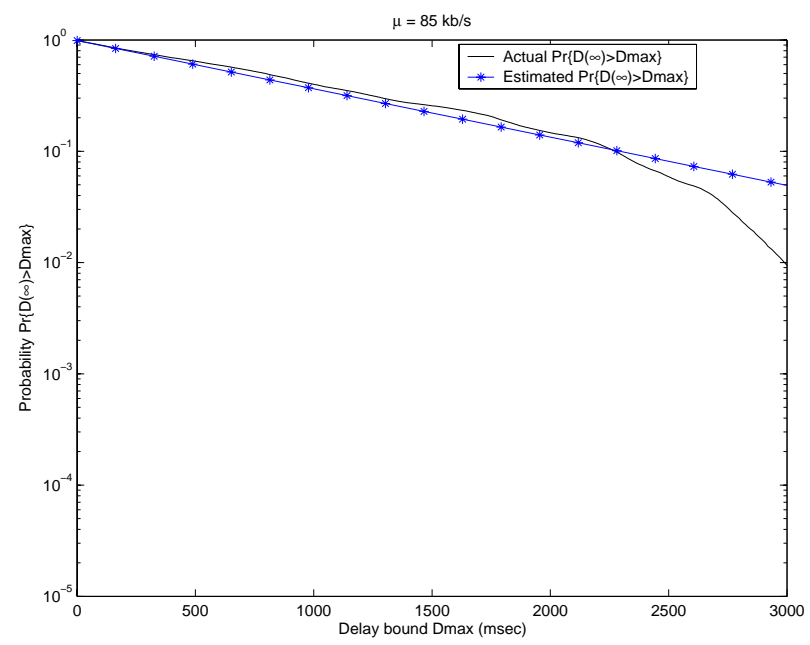

Figure 7: Actual and estimated delay-bound violation probability for $\mu=85 \mathrm{~kb} / \mathrm{s}$ and $N=1$.

Figure 6 shows the actual and estimated $\gamma_{N}$ vs. $N$ for various source rate $\mu$. The actual $\gamma_{N}$ is meant to be the $\gamma_{N}$ directly measured by Eqs. (39) through (42) for the case with $N$ sub-channels; the estimated $\gamma_{N}$ is obtained by Eq. (17), where $\eta^{(c)}(\mu)$ and $\beta^{(c)}(\mu)$ are estimated by (18) and (19), respectively. The figure demonstrates that 1) the actual $\gamma_{N}$ decrease exponentially with $N$, justifying the exponential relation in (17), and 2) the estimated $\gamma_{N}$ is close to the actual $\gamma_{N}$.

Figures 7 and 8 show the actual and estimated delay-bound violation probability $\operatorname{Pr}\{D(\infty) \geq$ $\left.D_{\max }\right\}$ vs. the delay bound $D_{\max }$, for various $N$ and $\mu=85 \mathrm{~kb} / \mathrm{s}$. The actual $\operatorname{Pr}\{D(\infty) \geq$ $\left.D_{\max }\right\}$ is obtained by directly measuring the queue; the estimated $\operatorname{Pr}\left\{D(\infty) \geq D_{\max }\right\}$ is obtained by Eq. (25), where $\left\{\beta^{(c)}(\mu), \eta^{(c)}(\mu), \theta_{1}(\mu)\right\}$ are estimated by (19), (18), and (39) through (42), respectively. The figures illustrate that the estimated $\operatorname{Pr}\left\{D(\infty) \geq D_{\max }\right\}$ agrees with the actual $\operatorname{Pr}\left\{D(\infty) \geq D_{\max }\right\}$. It is clear that as the number of sub-channels $N$ increases, $\operatorname{Pr}\left\{D(\infty) \geq D_{\max }\right\}$ decreases for a fixed $D_{\max }$. This indicates that frequency diversity improves the delay performance of a wireless channel. In [9], we showed how to utilize frequency diversity to improve delay performance.

Figure 9 shows the actual and estimated $\operatorname{Pr}\left\{D(\infty) \geq D_{\max }\right\}$ vs. the delay bound $D_{\max }$, for various $\mu$ and $N=4$. The actual and estimated $\operatorname{Pr}\left\{D(\infty) \geq D_{\max }\right\}$ are obtained by 


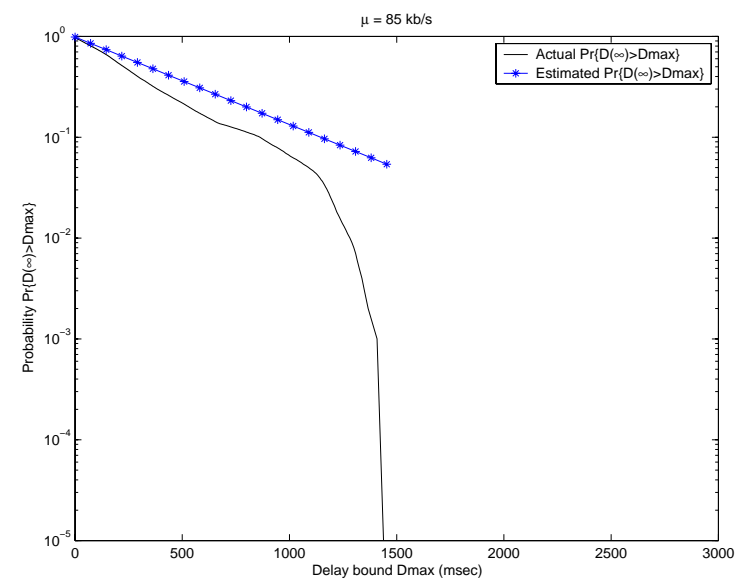

(a)

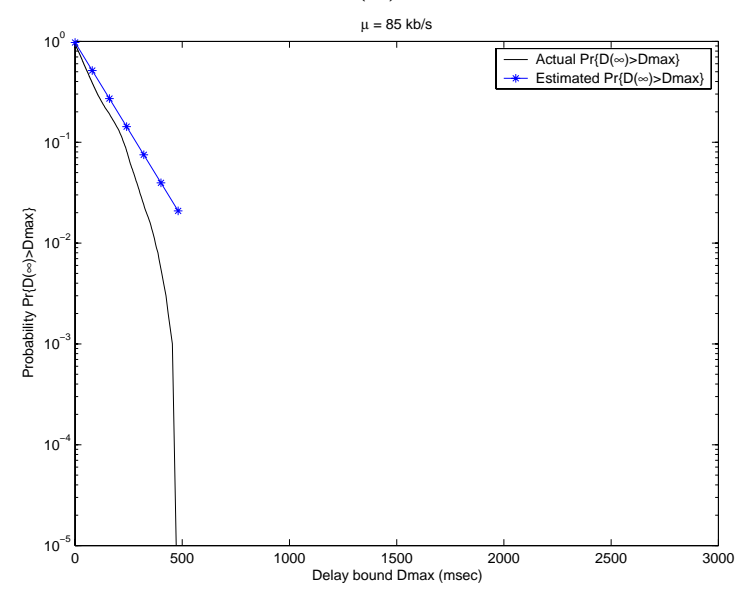

(c)

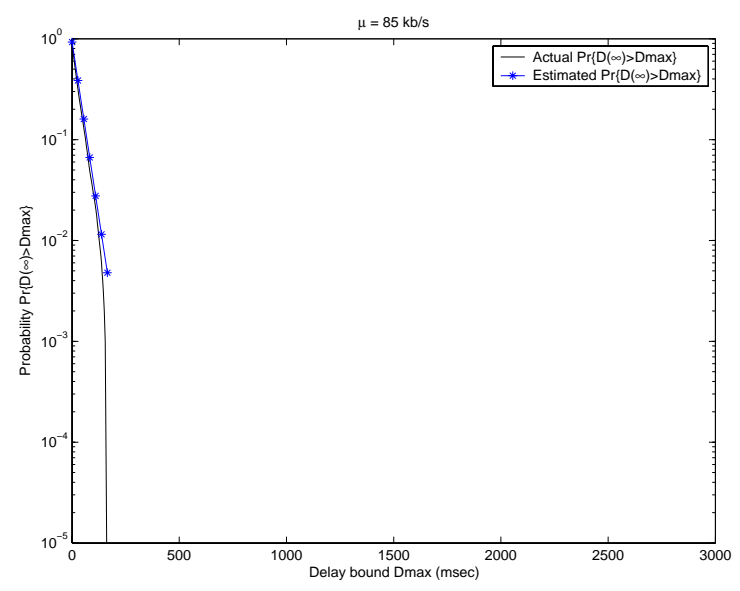

(e)

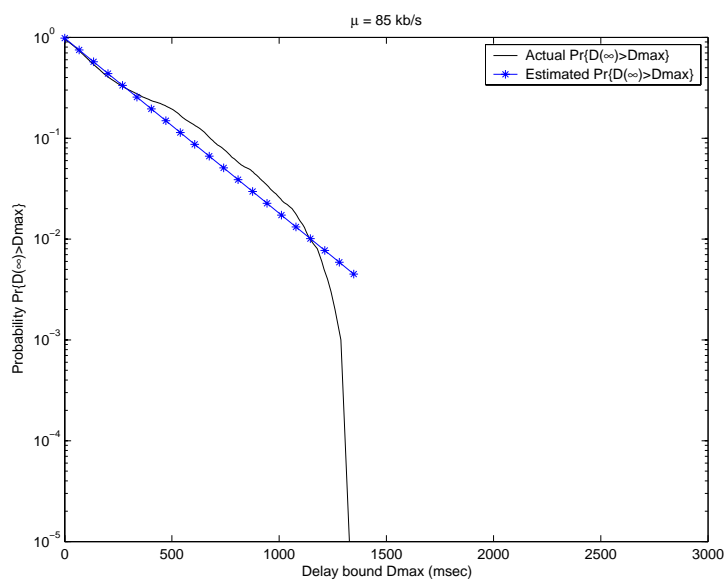

(b)

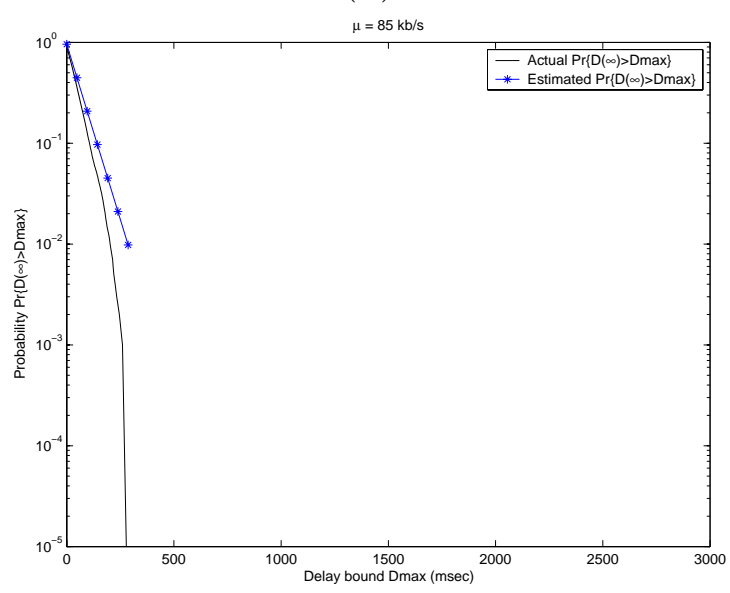

(d)

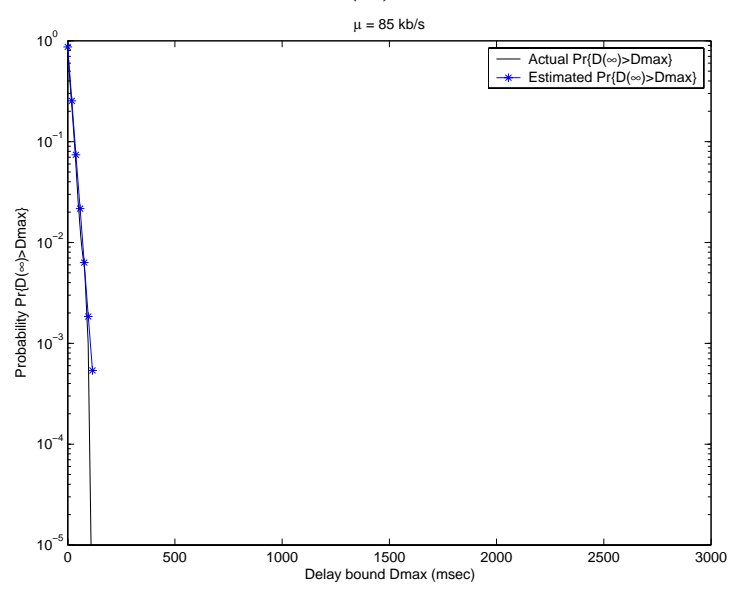

(f)

Figure 8: Actual and estimated delay-bound violation probability for $\mu=85 \mathrm{~kb} / \mathrm{s}$ and various $N$ : (a) $N=2$, (b) $N=4$, (c) $N=8$, (d) $N=16$, (e) $N=32$, and (f) $N=64$. 


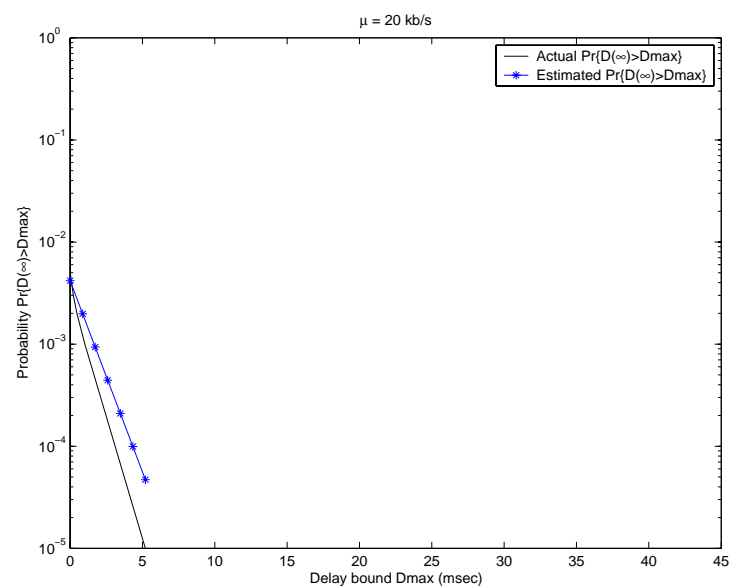

(a)

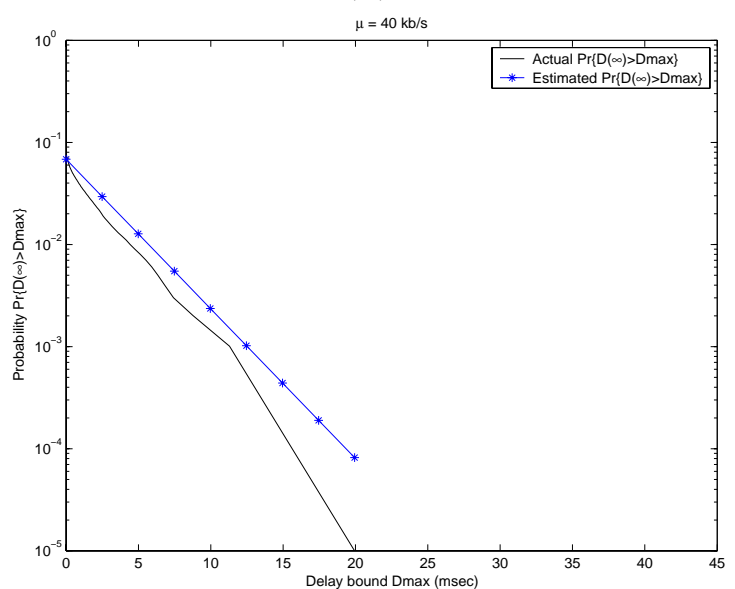

(b)

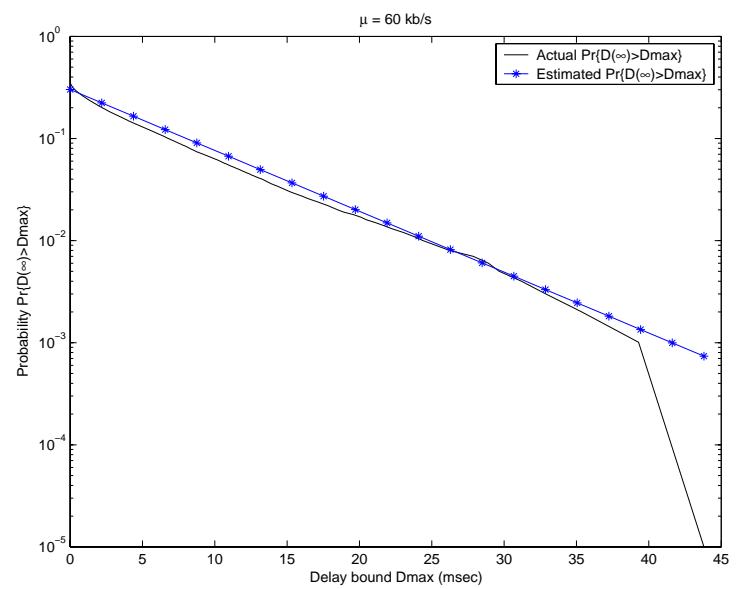

(c)

Figure 9: Actual and estimated delay-bound violation probability for $N=4$ channels: (a) $\mu=20 \mathrm{~kb} / \mathrm{s}$, (b) $\mu=40 \mathrm{~kb} / \mathrm{s}$, and (c) $\mu=60 \mathrm{~kb} / \mathrm{s}$. 


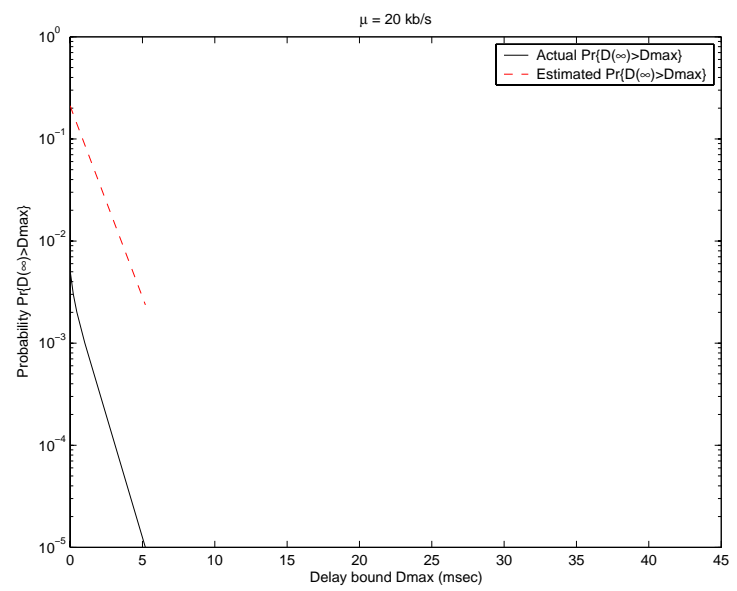

(a)

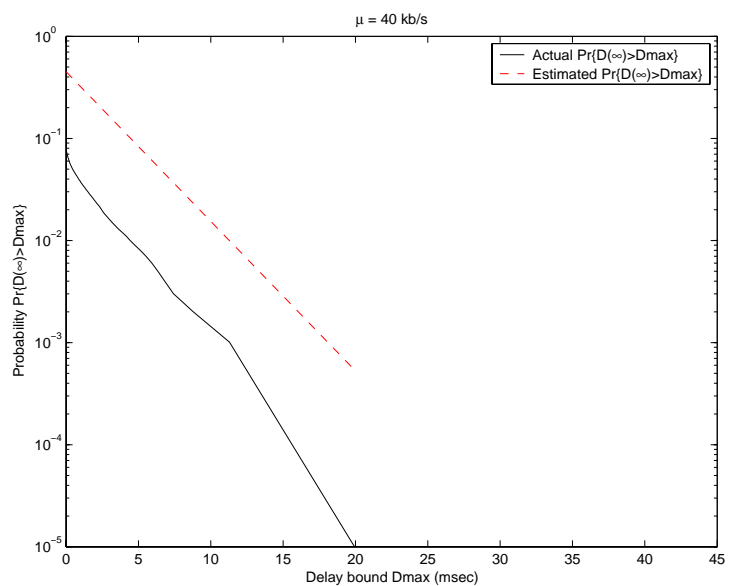

(b)

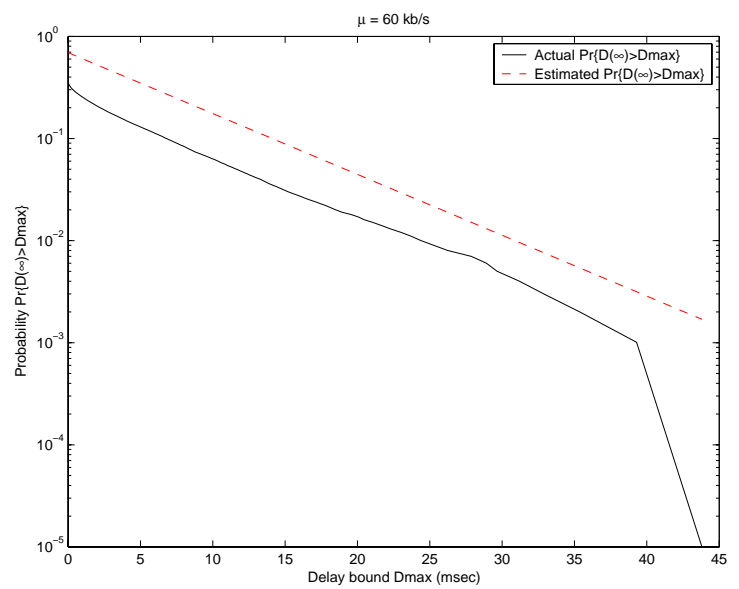

(c)

Figure 10: Actual and simply estimated delay-bound violation probability for $N=4$ channels: (a) $\mu=20 \mathrm{~kb} / \mathrm{s}$, (b) $\mu=40 \mathrm{~kb} / \mathrm{s}$, and (c) $\mu=60 \mathrm{~kb} / \mathrm{s}$. 
the same ways as Figure 8. Figure 9 indicates that the estimated $\operatorname{Pr}\left\{D(\infty) \geq D_{\max }\right\}$ gives good agreement with the actual $\operatorname{Pr}\left\{D(\infty) \geq D_{\max }\right\}$ for various data rates $\mu$.

Figure 10 shows the actual and estimated $\operatorname{Pr}\left\{D(\infty) \geq D_{\max }\right\}$ vs. the delay bound $D_{\max }$, for various $\mu$ and $N=4$, with a simplified estimation method. The actual $\operatorname{Pr}\{D(\infty) \geq$ $\left.D_{\max }\right\}$ is obtained by the same way as Figure 8. Different from Eq. (25), the estimated $\operatorname{Pr}\left\{D(\infty) \geq D_{\max }\right\}$ is obtained by a simplified estimate as below,

$$
\operatorname{Pr}\left\{D(\infty) \geq D_{\max }\right\}=\gamma_{1}(\mu) \times e^{-N \times \theta_{1}(\mu) \times D_{\max }},
$$

where $\gamma_{1}(\mu)$ and $\theta_{1}(\mu)$ are estimated by Eqs. (39) through (42) for the case with one subchannel. Compared with Figure 9, Figure 10 indicates that if $\gamma_{N}(\mu)$ in (16) is replaced by $\gamma_{1}(\mu)$, the estimated $\operatorname{Pr}\left\{D(\infty) \geq D_{\max }\right\}$ would be very conservative. Hence, the estimation of $\gamma_{N}(\mu)$ is necessary.

In summary, by estimating functions $\left\{\beta^{(c)}(\mu), \eta^{(c)}(\mu), \theta_{1}(\mu)\right\}$ and using Eq. (25), we can obtain the QoS $\operatorname{Pr}\left\{D(\infty) \geq D_{\max }\right\}$ for a frequency-selective fading channel, consisting of arbitrary $N$ i.i.d. sub-channels, with reasonable accuracy.

\section{Concluding Remarks}

In this paper, we proposed a link-layer channel model for frequency selective fading channels. The proposed model extends the effective capacity channel model we developed in [7]. Specifically, we utilize the duality between the distribution of a queue with superposition of $N$ i.i.d. sources, and the distribution of a queue with a frequency-selective fading channel that consists of $N$ i.i.d. sub-channels, to develop a model for frequency selective fading channels. Under the proposed model, a frequency selective fading channel is modeled by three EC functions, namely, $\left\{\beta^{(c)}(\mu), \eta^{(c)}(\mu), \theta_{1}(\mu)\right\}$; we also developed a simple and efficient algorithm to estimate these EC functions. Simulation results show that the actual QoS metric is closely approximated by the QoS metric predicted by the proposed EC channel model. The accuracy of our model can lead to efficient bandwidth allocation and QoS provisioning over wireless links. 


\section{Acknowledgment}

This work was supported by the National Science Foundation under the grant ANI-0111818.

\section{Appendix}

\section{Proof of Proposition 1}

Denote $r_{i}(t)$ the instantaneous channel capacity of sub-channel $i(i=1, \cdots, N)$ of the frequency-selective fading channel, at time $t$. Then for $u \geq 0$, we have

$$
\begin{aligned}
& \alpha_{N}(u) \stackrel{(a)}{=}-\lim _{t \rightarrow \infty} \frac{1}{u t} \log E\left[e^{-u \int_{0}^{t} r_{N}(\tau) \mathrm{d} \tau}\right] \\
& \stackrel{(b)}{=}-\lim _{t \rightarrow \infty} \frac{1}{u t} \log E\left[e^{-u \int_{0}^{t} \frac{1}{N} \sum_{i=1}^{N} r_{i}(\tau) \mathrm{d} \tau}\right] \\
& \stackrel{(c)}{=}-\lim _{t \rightarrow \infty} \frac{1}{u t} \log \left(E\left[e^{-u \int_{0}^{t} \frac{1}{N} r_{1}(\tau) \mathrm{d} \tau}\right]\right)^{N} \\
& =-\lim _{t \rightarrow \infty} \frac{1}{t \frac{u}{N}} \log E\left[e^{-\frac{u}{N} \int_{0}^{t} r_{1}(\tau) \mathrm{d} \tau}\right] \\
& \stackrel{(d)}{=} \quad \alpha_{1}\left(\frac{u}{N}\right)
\end{aligned}
$$

where (a) from (20), (b) since $r_{N}(t)=\frac{1}{N} \sum_{i=1}^{N} r_{i}(t)$, (c) since $r_{i}(t)(i=1, \cdots, N)$ are i.i.d., and (d) from (21). Then by $\alpha_{N}(u)=\alpha_{1}\left(\frac{u}{N}\right) \doteq \mu$, we have

$$
u=\alpha_{N}^{-1}(\mu)
$$

and

$$
\frac{u}{N}=\alpha_{1}^{-1}(\mu)
$$

Removing $u$ in (35) and (36) results in

$$
\alpha_{N}^{-1}(\mu)=N \times \alpha_{1}^{-1}(\mu)
$$


Thus, we have

$$
\begin{aligned}
\theta_{N}^{(c)}(\mu) & \stackrel{(a)}{=} \mu \alpha_{N}^{-1}(\mu) \\
& \stackrel{(b)}{=} \mu \times N \times \alpha_{1}^{-1}(\mu) \\
& \stackrel{(c)}{=} N \times \theta_{1}^{(c)}(\mu)
\end{aligned}
$$

where (a) from (22), (b) from (37), and (c) from (23). This completes the proof.

\section{Estimation of EC Functions $\{\gamma(\mu), \theta(\mu)\}$}

We briefly describe a simple algorithm to estimate the EC functions $\{\gamma(\mu), \theta(\mu)\}$ (see $[7]$ for details of derivation). Assume that the time-varying channel capacity process $r(n)$ is stationary and ergodic. For a given (unknown) fading channel and a given source rate $\mu$, we take measurements from the queue (see Fig. 4). Note that the queue is a only simulated queue, which is calculated based on the observed $r(n)$. First, take a number of samples, say $N$, over an interval of length $T$, and record the following quantities at the $n$th sampling epoch: $S_{n}$ the indicator of whether a packets is in service ${ }^{4}\left(S_{n} \in\{0,1\}\right), Q_{n}$ the number of bits in the queue (excluding the packet in service), and $T_{n}$ the remaining service time of the packet in service (if there is one in service). Then, compute the following sample means,

$$
\begin{aligned}
& \hat{\gamma}=\frac{1}{N} \sum_{n=1}^{N} S_{n}, \\
& \hat{q}=\frac{1}{N} \sum_{n=1}^{N} Q_{n},
\end{aligned}
$$

and

$$
\hat{\tau_{s}}=\frac{1}{N} \sum_{n=1}^{N} T_{n} .
$$

\footnotetext{
${ }^{4} \mathrm{~A}$ packet in service refers to a packet in the process of being transmitted.
} 
Finally, we obtain the estimate of $\theta(\mu)$ by

$$
\hat{\theta}=\frac{\hat{\gamma} \times \mu}{\mu \times \hat{\tau}_{s}+\hat{q}}
$$

Eqs. (39) through (42) constitute our algorithm for estimating the EC functions $\{\gamma(\mu), \theta(\mu)\}$. Note that, to get the functions $\gamma(\mu)$ and $\theta(\mu)$, we need to estimate $\gamma$ and $\theta$ for different source rate $\mu$.

\section{References}

[1] D. Botvich and N. Duffield, "Large deviations, the shape of the loss curve, and economies of scale in large multiplexers," Queueing Systems, vol. 20, pp. 293-320, 1995.

[2] G. L. Choudhury, D. M. Lucantoni, W. Whitt, "Squeezing the most out of ATM," IEEE Transactions on Communications, vol. 44, no. 2, pp. 203-217, Feb. 1996.

[3] H. Holma and A. Toskala, WCDMA for UMTS: Radio Access for Third Generation Mobile Communications, Wiley, 2000.

[4] Y. Y. Kim and S.-Q. Li, "Capturing important statistics of a fading/shadowing channel for network performance analysis," IEEE Journal on Selected Areas in Communications, vol. 17, no. 5, pp. 888-901, May 1999.

[5] T. S. Rappaport, Wireless Communications: Principles $\&$ Practice, Prentice Hall, 1996.

[6] B. Sklar, "Rayleigh fading channels in mobile digital communication systems Part I: characterization," IEEE Communications Magazine, vol. 35, no. 7, pp. 90-100, July 1997.

[7] D. Wu and R. Negi, "Effective capacity: a wireless link model for support of quality of service," IEEE Trans. on Wireless Communications, vol. 2, no. 4, pp. 630-643, July 2003.

[8] D. Wu and R. Negi, "Utilizing multiuser diversity for efficient support of quality of service over a fading channel," IEEE ICC'03, Anchorage, Alaska, USA, May 2003.

[9] D. Wu and R. Negi, "Downlink scheduling in a cellular network for quality of service assurance," Proc. IEEE Vehicular Technology Conference (VTC) Fall 2003, Orlando, Florida, USA, October 2003. 
[10] D. Wu, "Providing quality of service guarantees in wireless networks," Ph.D. Dissertation, Dept. of Electrical \& Computer Engineering, Carnegie Mellon University, Aug. 2003. Available at http://www. wu.ece.ufl.edu/mypapers/Thesis.pdf.

[11] Q. Zhang and S. A. Kassam, "Finite-state markov model for Rayleigh fading channels," IEEE Trans. Commun., vol. 47, no. 11, pp. 1688-1692, Nov. 1999.

[12] M. Zorzi, R. R. Rao, and L. B. Milstein, "Error statistics in data transmission over fading channels," IEEE Trans. Commun., vol. 46, no. 11, pp. 1468-1477, Nov. 1998.

[13] M. Zorzi, A. Chockalingam, and R. R. Rao, "Throughput analysis of TCP on channels with memory," IEEE Journal on Selected Areas in Communications, vol. 18, no. 7, pp. 1289-1300, July 2000 . 